\title{
Institutionenökonomische Perspektiven im Kontext der Reorganisation subnationaler Gebietskörperschaften
}

\author{
Walter Thomi, Halle (Saale)
}

\section{Einleitung}

1.1 Wissenschaftliche Diskurse zur Dezentralisierung Diskurse um Möglichkeiten und Grenzen von Dezentralisierung bilden einen festen Bestandteil entwicklungspolitischer Diskussionen seit ihren professionalisierten Anfängen in den 50er Jahren (CONYERS 1984). Immer wieder beschäftigten sich vor allem die Disziplinen der Verwaltungswissenschaft, des Verfassungsund Verwaltungsrechts, der Ökonomie, Politologie und Soziologie, aber auch der Raum- und Regionalplanung sowie gelegentlich auch der Geographie mit den Ursachen, Möglichkeiten und Wirkungen von Dezentralisierung aus ihren jeweils fachspezifischen Perspektiven heraus (vgl. Tном⿰ et al. 2000).

Während Ökonomen Dezentralisierung ganz wesentlich als eine Option zur Verbesserung der Allokationseffizienz des Staates bei Bereitstellung öffentlicher Güter unter Annahme von regional differierenden Präferenzen ansahen (vgl. u.a. Musgrave \& Musgrave 1973, OATES 1972), konzentrierten sich Verwaltungswissenschaftler mehr auf die Umsetzung des Gestaltungsprinzips der Subsidiarität in zu dezentralisierenden Verwaltungsstrukturen und Verwaltungsabläufen (vgl. u.a. Simon, Stockmayer \& Fuhr 1993, Bodemer 2000). In den Sozialwissenschaften wurden häufig herrschaftssoziologische Aspekte der Dezentralisierung diskutiert (vgl. u.a. Slater 1989, Agrawal 1999). Raum- und Regionalplaner sahen vor allem in den 80er Jahren Dezentralisierung als Option einer mehr bedarfsgerechten, weil bürgernahen Planung und Entwicklung (vgl. u.a. RondinelLi \& CHEEMA 1983), und in der Geographie wurde über Dezentralisierung zumeist im Rahmen einer mehr länderbezogenen Perspektive berichtet (vgl. u.a. Holtкamp 1993, Thом⿰ 1999).

Im Ergebnis präsentierten sich eine Fülle von Fallstudien, die eine Vielzahl von Dezentralisierungsaktivitäten aus einer eben solchen Vielzahl von Ländern vorstellten, ohne dass es in der Regel gelang, sich aus dem spezifischen normativen Fachkontext zu lösen und eine theoretisch konsistente Interpretation zu entwickeln (vgl. BEIER 1995).

\subsection{Dezentralisierungstrends in den Entwicklungsländern}

Die akademische Auseinandersetzung mit den Möglichkeiten und Grenzen der Dezentralisierung in Entwicklungsländern stand natürlich auch in engem
Zusammenhang mit diesbezüglichen Aktivitäten in den entsprechenden Ländern. So lassen sich verstärkte Reformaktivitäten im Rahmen der Dekolonisierung in den 50er und 60er Jahren feststellen, die jedoch im Ergebnis eher eine starke Zentralisierungstendenz aufwiesen. Diese ergab sich aus den damaligen Präferenzen für einen starken Zentralstaat als Entwicklungsträger und aus der Notwendigkeit der Überwindung der regionalen, meist ethnischen Partikularismen durch «Nationbuilding» (vgl. u.a. RoNDINELLI 1999). Eine zweite Dezentralisierungswelle zeichnete sich in den 70er und 80er Jahren ab. Diese stand in Zusammenhang mit der Krise des postkolonialen Entwicklungsstaats, dessen enttäuschende Ergebnisse und hohe Kosten häufig eine Reorganisation der staatlichen Verwaltungen mit den Zielen der Kostenreduzierung und Herrschaftssicherung erforderten, wobei interner (wirtschaftliche und politische Krisen) und externer Handlungsdruck (Verschuldung) sich in den betroffenen Ländern jeweils sehr unterschiedlich artikulierten (vgl. WunSCH \& Oluwu 1995). In den 90er Jahren wurde der Reformdruck noch einmal verstärkt durch die weltweiten Demokratisierungstendenzen aber auch durch den mit der Globalisierung in Zusammenhang stehenden Bedeutungsverlust nationalstaatlicher Regulationsrahmen und der damit in Verbindung stehenden Bedeutungszunahme subnationaler Regulationsrahmen (vgl. Тномі 2001). Mit Dezentralisierungsaktivitäten in mehr als 75 Ländern erreicht das Phänomen in dieser Zeit einen neuen Höhepunkt, so dass einige Autoren schon vom Zeitalter der Dezentralisierung sprechen (vgl. FuHR 1999).

\subsection{Neue Ansätze zur Dezentralisierung}

Im Kontext dieser Entwicklungen und damit verbundener Erfahrungen wird auch der verwaltungstechnisch reduzierte Dezentralisierungsbegriff erweitert. Es geht nicht mehr nur um die Verschiebung von politischen, administrativen und fiskalischen Verantwortlichkeiten auf vorgelagerte oder rangniedere Verwaltungsebenen, sondern um eine Reorganisation öffentlicher Aufgaben unter Einbeziehung des privaten und zivilgesellschaftlichen Bereichs (vgl. Simon 2000). Eine zentrale Bedeutung in dieser Diskussion spielt der Begriff «Good Governance», der nicht nur die Steuerungskapazitäten und Fähigkeiten des Staates, sondern auch die der Gesellschaft mit einbezieht (vgl. Adam 2000). Neben den empirischen Erfahrungen der Reformprojekte sind es vor allem neuere Ansätze aus der Ökonomie, die für einen gegenüber früheren Diskussionen erweiterten Diskussionsrah- 
men von Dezentralisierung sorgen. Dabei handelt es sich insbesondere um die neue Institutionenökonomie, die in den 90er Jahren aus ihrem engen fachlichen Diskussionkontext heraustrat und zunehmend auch in den Nachbarwissenschaften Beachtung fand. Es geht hierbei nicht um ein bereits geschlossenes neues Gebäude wirtschaftswissenschaftlicher Logik, sondern eher um eine «Großbaustelle» einer Vielzahl von Autoren, die sich mit unterschiedlichen Schwerpunktsetzungen darum bemühen, die Bedeutung, Funktionsweise und Entwicklung institutioneller Ordnungen für den Wirtschaftsprozess zu erklären (vgl. RichTER \& FURUBOTON 1999).

\section{Ausgewählte Aspekte der Neuen Institutionenökonomie}

Es ist das Ziel der nachfolgenden Ausführungen, einige für die Dezentralisierungsproblematik besonders interessante Grundgedanken der Ansätze zur Neuen Institutionenökonomie, kurz NIÖ-Ansätze genannt, vorzustellen und dahingehend zu diskutieren, ob sie eine verbesserte Analyse und Gestaltung von Dezentralisierungsprozessen erlauben. Diese Diskussion wird in vier Abschnitten organisiert. Zunächst werden einige Grundannahmen zur gesellschaftlichen Entwicklung vorgestellt, denen eine Skizzierung der Staatstheorie der NIÖ folgt. Der Zusammenhang von Staat und Entwicklung aus Sicht der NIÖ ist Gegenstand der nachfolgenden Diskussion. Abschließend wird auf die Bedeutung des Ansatzes zur Analyse und Gestaltung von Dezentralisierungsprozessen eingegangen.

\subsection{Institutionen und Regulation}

Gesellschaftliche Entwicklung lässt sich abbilden als ein Prozess zunehmender Spezialisierung, in dem Individuen oder auch Gruppen sich (meist auf der Basis technischer oder sozialer Innovationen) auf spezifische Tätigkeitsbereiche konzentrieren. Dieser Prozess einer zunehmenden Arbeitsteilung oder Spezialisierung bzw. die damit verbundenen Produktivitätssteigerungen verursachen ein wirtschaftliches Wachstum, welches seinerseits wieder die Grundlage für weitere Spezialisierungen und damit Entwicklungen darstellt. Nun handelt es sich dabei aber nicht lediglich um eine Frage der Verfügbarkeit von Technologien und Ressourcen, sondern auch um einen gesellschaftlichen Prozess, dessen Dynamik an spezifische, über die unmittelbare Produktion hinausgehende Voraussetzungen gebunden ist: Mit dem Prozess der Spezialisierung ist unmittelbar eine Zentralisierung von Funktionen (Tätigkeiten) sowie gleichzeitig eine ökonomische und soziale Differenzierung der beteiligten Akteure verbunden. Weiterhin liegt es im Wesen der Spezialisierung, dass mit ihr die Notwendigkeit des Austausches oder - abstrak- ter formuliert - die Notwendigkeit zur Integration und Interaktion entsteht. «Spezialisten» können in einem gesellschaftlichen Ganzen nur wirken, wenn die notwendigen Transaktionen bzw. Austauschprozesse zur Versorgung aller Mitglieder organisiert sind. Hierzu bedarf es akzeptierter und durchsetzbarer Regulationen, die Transaktionen zur ausreichenden Verteilung aller notwendigen Güter in einer Weise gewährleisten, dass die spezialisierten Produzenten ihre Aktivitäten dauerhaft wahrnehmen können (vgl. Abb.1). Die Institutionenökonomie spricht in diesem Zusammenhang von der Notwendigkeit der Herausbildung von Institutionen und meint damit ein System formungebundener (informeller) und formgebundener (formeller) Regeln, die den Zweck verfolgen, individuelles Verhalten in eine gewünschte Richtung $\mathrm{zu}$ lenken. NORTH (1992) betont in diesem Zusammenhang die Bedeutung dieser Regeln für die Reduzierung von Unsicherheit und interpretiert sie als eine Art Anreizstruktur der Gesellschaft. Werden diese Regeln von spezifischen Personen vertreten, spricht man von Organisationen.

Ohne weitere Details zu diskutieren, ergeben sich aus dem bisher skizzierten Bild zwei Konsequenzen:

1. Je weiter der Grad an Spezialisierung in einer Gesellschaft voranschreitet, desto mehr Transaktionen werden notwendig. Damit verbunden ist ein zunehmend komplexer werdendes Regelwerk von Institutionen und diese repräsentierenden bzw. überwachenden Organisationen (vgl. Abb. 1).

2. Wirtschaftliches Wachstum wird damit auch abhängig von der Herausbildung der für komplexere Transaktionen notwendigen Regeln (Institutionen) und deren Einhaltung überwachende Organisationen. Diese beeinflussen ihrerseits natürlich auch die Dynamik der weiteren Spezialisierung und Arbeitsteilung. In diesem Sinne ist wirtschaftliches Wachstum nicht nur im technologischen, sondern auch im institutionellen Sinne als pfadgebundenes Phänomen zu bezeichnen, da die gesellschaftlichen Anreizstrukturen die Dynamik des Prozesses zumindest beeinflussen, wenn nicht gar steuern (vgl. Frenkel \& Hemmer 1999: 307-320).

Damit gelingt es der Institutionenökonomie, wirtschaftliches Wachstum aus dem engen Zusammenhang von Produktionsfaktorkombinationen zu lösen und die Bedeutung der gesellschaftlichen Institutionen und Organisationen für diesen Prozess zu begründen.

\subsubsection{Das Paradigma des Sozialen in der NIÖ}

Wie wird nun die Entstehung von kollektiven Regeln individuellen Handelns abgeleitet? Den gesellschaftlichen Akteuren wird ganz in der Tradition der Neoklassik eine individuelle Nutzenmaximierung unterstellt (methodischer Individualismus), aus der sich dann allerdings kein harmonisches Ganzes ergibt, sondern Konstellationen gegenseitiger Beeinträchtigung, 


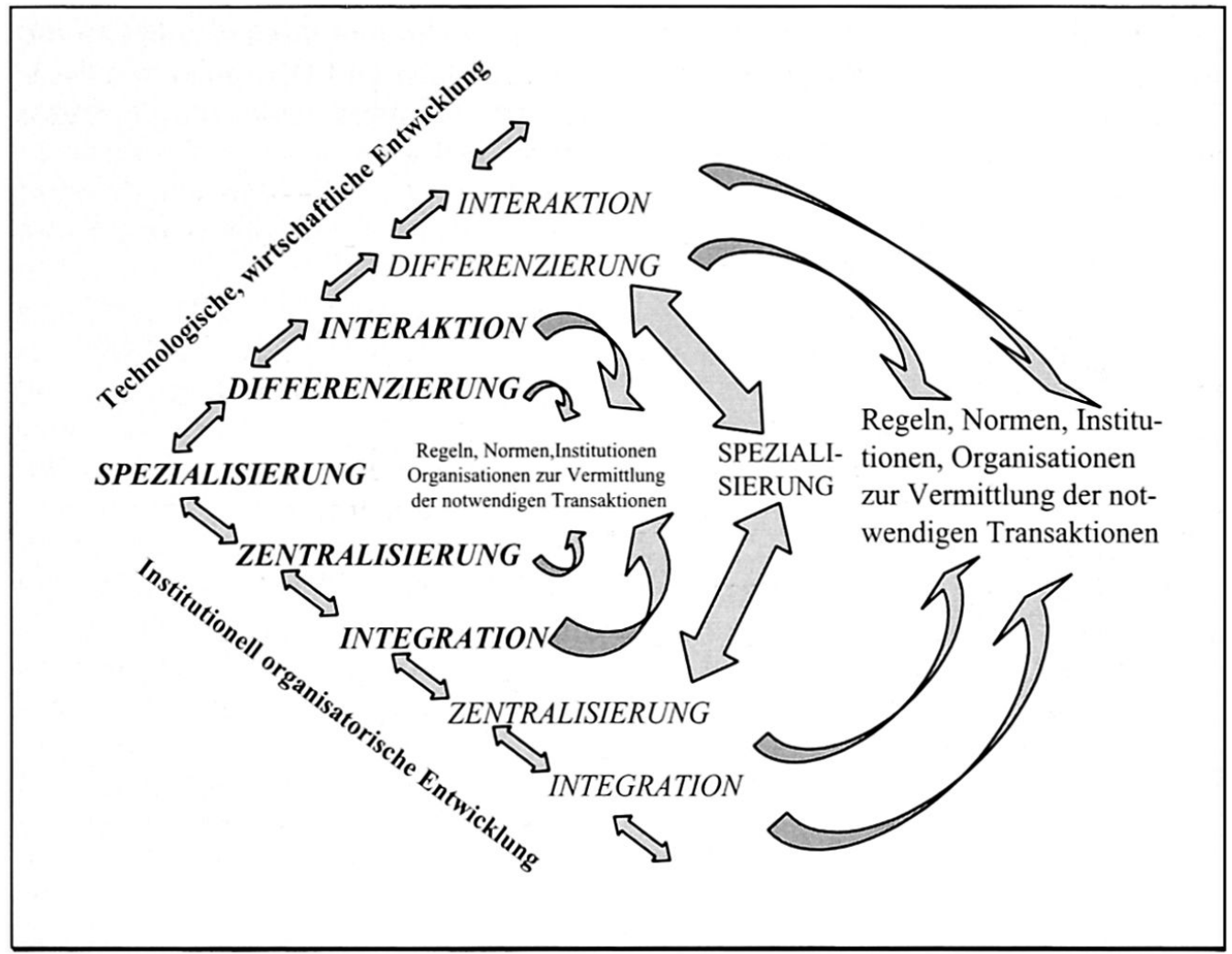

Abb. 1: Interdependenz von wirtschaftlicher und institutioneller Entwicklung

Interrelation between economic and institutional development Interdépendance du développement économique et institutionnel

Quelle: Тномі 2001

die nur durch kollektive/soziale Lösungen zu beheben sind. Damit verbunden ist stets eine Mehrung der Wohlfahrt aller Beteiligten, wenn sich diese an die Regeln halten. Die Herausbildung dieses kollektiven Rationalismus ist, wie mit Hilfe des Paradigmas vom Gefangenendilemma (Prisoners' Dilemma) gezeigt, nicht zwangsläufig und hängt von der Richtung der kollektiven Lernprozesse ab. Mit Hilfe des Paradigmas wird das gesellschaftliche Dilemma des Menschen in einer Weise dargestellt, dass die Vorteile einer gemeinsamen Strategie auf Vertrauen, Einsicht und Unterdrückung des sogenannten «natürlichen» Eigennutzes beruhen.

Zur Verdeutlichung der Problemstellung lässt sich das Paradigma mit Hilfe von zwei Weizenproduzenten (A und B ) darstellen. Zur individuellen Nutzenmaximierung besteht für beide die Option sich entweder auf den eigenen Anbau zu konzentrieren oder aber zusätzlich Weizen beim Nachbarn zu stehlen (vgl. Abb. 2).

Die in Klammern angegeben Werte stellen die Erträge der Akteure bei der jeweils gewählten Strategie dar. Zelle 3 wird als «Naturzustand» angesehen. Beide Akteure stehlen, und es ergibt sich infolge der Bewachungs- und Raubkosten ein insgesamt niedriger
Ertrag (16). Stiehlt nur einer der Produzenten («Trittbrettfahrer»), so kann dieser seinen individuellen Ertrag auf Kosten des anderen erhöhen (Zelle 2 und 4). Durch diese Option wird das System tendenziell instabil, denn sie kann immer wahrgenommen werden. Die Erreichbarkeit von Zelle 1 mit dem maximalen Ertrag (19) wird davon abhängig sein, ob es gelingt, Regeln und damit Anreiz- bzw. Sanktionssysteme zu schaffen, die eine Eintrittswahrscheinlichkeit der Zelle

\begin{tabular}{|l|ll|ll|}
\hline \multicolumn{1}{|c|}{ B } & \multicolumn{2}{|c|}{ stiehlt nicht } & \multicolumn{2}{c|}{ stiehlt } \\
\hline stiehlt nicht & $\mathbf{1}$ & $(10 / 9)$ & $\mathbf{4}$ & $(7 / 11)$ \\
\hline stiehlt & $\mathbf{2}$ & $(12 / 6)$ & $\mathbf{3}$ & $(8 / 8)$ \\
\hline
\end{tabular}

Abb. 2: Diebstahloption als Variante des Gefangenendilemmas

Stealing as prisoners' dilemma

Le vol, une variante du dilemme des prisonniers

Quelle: Mueller 1995:10 
2 und 4 erheblich reduzieren (vgl. Mueller 1995). Die Herausbildung solcher Regeln lässt sich nun nach den Prinzipien der evolutionären oder konstruktivistischen Rationalität mit Hilfe der Theorie der unsichtbaren Hand erklären (HAYEK 1973).

Wichtig erscheint, dass es sich in dieser Konstruktion lediglich um Optionen handelt, die sich nicht notwendigerweise realisieren müssen. Entwicklung im Sinne einer erhöhten Wohlfahrt (Zelle 1) wird aber nur erreicht, wenn es gelingt, ein die Akteure in diesem Sinne steuerndes Anreizsystem zu etablieren. Es erscheint weiterhin einleuchtend, dass zur Erhöhung der Verbindlichkeit dieser Regeln (Institutionen) unter Umständen Organisationen, also spezialisierte Personen hilfreich sein können, wodurch allerdings auch zusätzliche Kosten entstehen.

An dieser Stelle wird eine gewisse geistige Nähe des NIÖ-Ansatzes zu den Regulationstheorien (BOYER \& SaILlard 1995) deutlich, die zwar mehr im Sinne einer Klassifikation von zeitlich begrenzt stabilen Gesellschaftsformationen ausgehen, die aber auch den Zusammenhang von Produktion und damit korrespondierenden institutionellen Formen zur Regulation thematisieren (vgl. KRÄTKE 1996).

\subsection{Transaktionskosten und Staat}

In ihrem ökonomischen Kern dienen gesellschaftliche Anreizstrukturen der Regulation von Verfügungsrechten. Diese werden in der Property Rights-Analyse als gesellschaftlich akzeptierte Rechte über materielle und immaterielle Objekte definiert (SCHÜLLER 1983). Schon die Aufrechterhaltung von Verfügungsrechten erfordert (Überwachungs-) Kosten. Die Veränderung von Handlungsrechten durch Transaktionen lässt zusätzliche (Transaktions-) Kosten für die Akteure entstehen. Darunter werden ganz wesentlich die Kosten der Geschäftsanbahnung (z. B. Informationskosten) und der Überwachung bzw. Einhaltung verstanden. Es erscheint nun evident, dass die Schaffung von Institutionen, inklusive der sie überwachenden Organisationen, die individuellen Transaktionskosten erheblich senken können. Solche für alle Mitglieder der Gesellschaft geltenden Institutionen können auch als öffentliche Güter (Nicht-Ausschliessbarkeit und keine Nutzungskonkurrenz) bezeichnet werden. Dazu zählen nicht nur immaterielle Güter (Sicherheit, Recht, etc.), sondern auch die diese unterstützende materielle Infrastruktur (Schulen, Strassen, etc.). Die Notwendigkeit öffentlicher Güter entsteht mit zunehmender gesellschaftlicher Komplexität als deren Resultat und als Voraussetzung weiterer Entwicklungen.

Während nun in kleinen überschaubaren Gesellschaften die Bekanntheit und das damit verbundene Vertrauen zur Regulation der gesellschaftlich notwendigen Transaktionen ausreichten (Haushalte, Clans), stoßen diese Regulationsformen ab einem bestimmten gesellschaftlichen Spezialisierungs- und Differenzie- rungsgrad an ihre Grenzen, da sich die Akteure nicht mehr kennen und Vertrauen gesellschaftlich konstruiert werden muss. Es leuchtet ein, dass damit Kosten verbunden sind.

Aus diesem Problemkreis heraus entsteht nun der Staat als hochzentralisierte Regulationsform, dessen Wesen und Aufgabe darin besteht, durch Bereitstellung von öffentlichen Gütern die individuellen Transaktionskosten der Akteure zu senken. Der Staat wird als Organisation definiert, dessen Primärfunktion auf die Schaffung und Einhaltung von solchen Institutionen zielt, die eine Senkung der individuellen Transaktionskosten ermöglichen und damit gleichzeitig die Wohlfahrt insgesamt erhöhen. Da dem Staat zur Durchsetzung von Schutz und Gerechtigkeit in der Regel das Gewaltmonopol zukommt, verfügt er auch über ein Rentenmonopol zur Finanzierung seiner Ausgaben, d.h. er kann Abgaben (Steuern) von seinen Mitgliedern erzwingen.

Den Repräsentanten des Staates (Herrscher oder Regierung) wird nun der gleiche Eigennutz wie allen anderen Individuen unterstellt, d.h. es wird eine Orientierung staatlichen Handelns auf Maximierung der Monopolrente angenommen.

Obwohl der Staat die oberste Autorität auf seinem Territorium bildet, ist er trotzdem nicht völlig unabhängig. Zum einen gibt es rivalisierende Nachbarstaaten (Intervention), aber fast immer auch lokale Herrschaftsrivalen (Konkurrenz). Darüber hinaus benötigt der Staat ein Mindestmaß an interner Legitimation und daraus resultierender freiwilliger Kooperation. Ansonsten würden die Überwachungskosten aber auch die Bereitstellungskosten der öffentlichen Güter zu hoch bzw. diese könnten nicht mehr in ausreichendem Maße produziert werden (vgl. RichTER \& FurUBoton 1999). Die Bürger würden unzufrieden werden und beginnen, über die Absetzungskosten des «Herrschers» nachzudenken oder aber, wenn möglich, ihre «ExitOption» wahrzunehmen. Insgesamt sind dem Staat solchermaßen im Hinblick auf die Abschöpfung der Monopolrente gewisse Grenzen gesetzt.

Die grundlegenden Regulationen zwischen Staat und Bürger werden in der Verfassung festgelegt. Aus der Perspektive der NIÖ stellt die Verfassung ein Regelbzw. Anreizsystem dar, um die Beziehungen zwischen Staat und Bürgern zu regulieren, wobei die Struktur der Verfügungsrechte der Staatsangehörigen von entscheidender Bedeutung ist. Dabei kann es sich um eine geschriebene oder auch ungeschriebene Verfassung handeln. Sie sollte im Sinne von sich selbst durchsetzenden Verträgen so gestaltet sein, dass es für alle Beteiligten längerfristig lohnender ist, die darin enthaltenen Regeln zu respektieren und einzuhalten, als diese zu brechen (Senkung der Überwachungskosten). In der realen Ausgestaltung können Verfassungen ebenso wie Staaten sehr unterschiedliche Formen annehmen, was wesentlich auch durch die regionalen 
Besonderheiten mitbestimmt wird. Höhe und Verwendung der staatlichen Monopolrente variieren ebenfalls.

Der Staat wird je nach Staatsform vertreten durch einen Herrscher oder eine Regierung (Exekutive) sowie eine Verwaltung (Legislative). Diese Vertretungsfigur lässt sich in der NIÖ mit Hilfe des «Prinzipal-Agent-Modells» abbilden. Die Grundstruktur der Prinzipal-Agent-Beziehung (PAB) ist einfach: Der Prinzipal (Vertretener) benötigt zur Umsetzung von Aufgaben einen Agenten (Vertreter). Zwischen beiden besteht eine Informationsasymmetrie, derzufolge der Prinzipal wenig über die konkrete Arbeitssituation des Agenten weiß und diese auch nicht kontrollieren kann. Er kann seinen Agenten nur über die erzielten Ergebnisse bewerten, über deren Ursachen er nur durch den Agenten informiert wird. Der Vertrag bzw. die Regeln zwischen beiden müssen aus Sicht des Prinzipals so gestaltet werden, dass es für den Agenten sinnvoll wird, im Sinne der vom Prinzipal gewünschten Ergebnisse zu handeln.

Diese PAB wird im Staatskontext mehrfach verwendet. Zum einen kann man Wähler als Prinzipale betrachten und die Regierung als Agenten. Hier ergibt sich das Problem, wie sich die Wähler gegen opportunistisches Verhalten ihrer Regierung schützen können, was in der «Public Choice Theorie» thematisiert wird (vgl. MülleR 1995).

Zum anderen lässt sich $\mathrm{PAB}$ auch für die Beziehung zwischen Verwaltung (Agent) und Regierung (Prinzipal) nutzen. So könnte z.B. die Verwaltung die Monopolrenten der Regierung in Teilbereichen vergeuden. Es stellt sich dann die Frage nach einer adäquaten Gestaltung der Anreizstrukturen zur Reduzierung dieser Probleme. Bereits hier wird die konkrete Anwendbarkeit auch für Fragen der Dezentralisierung deutlich, denn natürlich kann man Dezentralisierung auch unter dem Gesichtspunkt einer Veränderung von Anreizsystemen analysieren und wird wahrscheinlich wesentlich mehr erfolgsrelevante Fragestellungen erkennen, als das mit Hilfe von «traditionellen Ansätzen» möglich ist (vgl. Weltbank 1997 \& Fuhr 1999).

\subsection{Staat und Entwicklung}

Generell wurde bereits auf die wichtige Rolle der die Transaktionskosten senkenden Regeln und die Bedeutung des Staates in diesem Kontext hingewiesen. $\mathrm{Ab}$ einem spezifischen Komplexitätsstadium (Differenzierungsgrad) können die wirtschaftlichen Transaktionen nur abgewickelt werden, wenn ein zentralstaatlicher Regulationsrahmen besteht. In diesem Sinne ist er eine notwendige Voraussetzung für Entwicklung, weil erst durch ihn die für langfristige Verträge/Investitionen/ Transaktionen notwendigen öffentlichen Güter dauerhaft bereitstellt werden können. Insofern bleiben auch die ökonomischen Entwicklungsmöglichkeiten von nicht zentralstaatlich organisierten Gesellschaften begrenzt. Allerdings ist der Staat keine hinreichende Bedingung für Entwicklung. Aufgrund der vorgestellten Annahmen des Eigennutzes auch der staatlichen Vertreter besteht für den Staat tendenziell das Risiko, eine zu hohe Monopolrente abzuschöpfen. Dies geschieht immer dann, wenn der Staat die durch ihn selbst entstandenen individuellen Transaktionskostenersparnisse selbst abschöpft, wodurch die Motivation für zusätzliche wirtschaftliche Transaktionen bei den Bürgern zumindest stark gedämpft, wenn nicht gar unterbunden wird. In einem solchen Fall kann von Ausbeutung oder Missbrauch der staatlichen Monopolsituation (Staatsversagen) gesprochen werden. In dem $\mathrm{Maße}$, wie der Staat den wirtschaftlichen Akteuren Ressourcen entzieht und gleichzeitig seine öffentlichen Güter und Dienstleistungen nicht mehr adäquat, d.h. transaktionskostensenkend bereitstellen kann, ergibt sich eine insgesamt negative Entwicklungssituation. Die Produktivität der Wirtschaft verschlechtert sich, weil der Staat nicht die notwendigen öffentlichen Güter bereitstellen bzw. unterhalten kann, wodurch sich wiederum die wirtschaftliche Situation und damit die Ressourcenbasis des Staates verschlechtert.

In einer statischen Betrachtung stellen deshalb Höhe und Verwendung der staatlichen Monopolrente entscheidende Parameter zur Beurteilung von staatlicher Effizienz und Effektivität dar, wobei die Aufgabenbereiche staatlicher Regulation sich nicht absolut bestimmen lassen und einem permanenten politischen Bewertungsprozess unterliegen. So wurde z.B. in Deutschland die Einführung der staatlichen Altersversicherung (Rentenregulation) im 19. Jahrhundert als große Errungenschaft mit weitreichenden Folgen bis in die Gegenwart gefeiert. Heute wird in diesem Zusammenhang aber über privatwirtschaftliche Regulationsformen diskutiert. Es hängt also von der spezifischen historischen Situation und den verfügbaren kollektiven Erfahrungen und Technologien ab, in welchem Maße der Staat oder andere gesellschaftliche Organisationsformen wie der Markt oder die Zivilgesellschaft die zur gesellschaftlichen Reproduktion notwendigen Güter und Dienstleistungen bereitstellen. In diesem Sinne verfügt jede Gesellschaft über eine spezifische Regulationsmatrix, welche in ihrer Gesamtheit die Bereitstellung der zur gesellschaftlichen Reproduktion notwendigen öffentlichen und privaten Güter reguliert. Diese lassen sich als eine Art Kontinuum darstellen. Einen Pol bilden die öffentlichen Güter, denen am anderen Ende private Güter gegenüberstehen. Dazwischen gibt es die sogenannten Waren, die über den Markt reguliert werden (vgl. Abb. 3). Die Bereitstellung dieser Güter wird über die vier Regulationsformen Staat, Zivilgesellschaft (partikulare Interessensverbände), Markt und Haushalte organisiert. In welchem Maße der Staat bzw. die anderen Regulationsformen welche Güter bereitstellen, hängt 


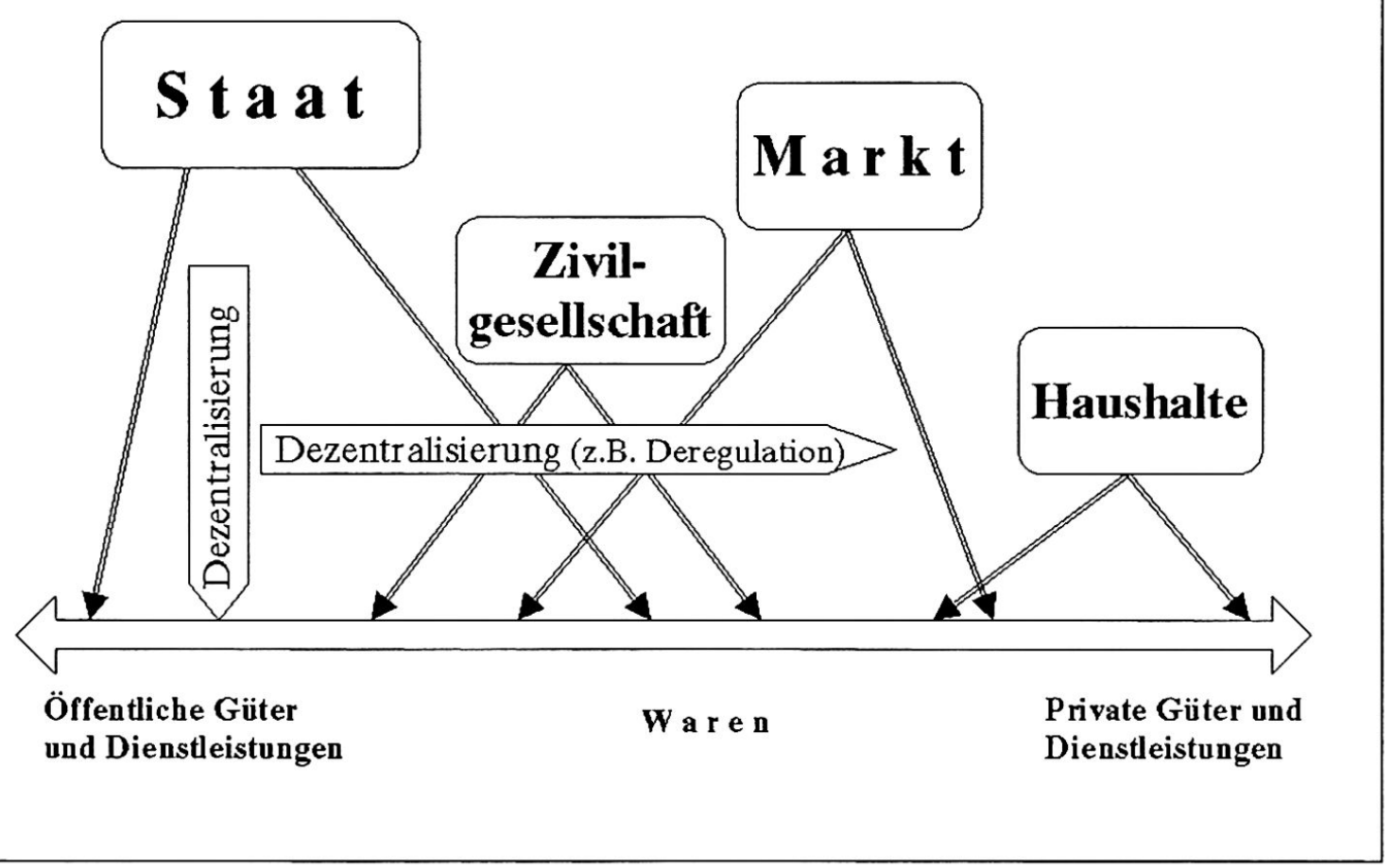

Abb. 3: Wirkungsgefüge von Dezentralisierung innerhalb der gesellschaftlichen Regulationsmatrix Impact of decentralisation within the context of a society's regulative matrix Impact de la décentralisation dans la matrice sociale de régulation

Quelle: Tномі 2001

vom gesellschaftlichen Diskurs und den verfügbaren Technologien ab und ist einem steten Wandel unterworfen. Abbildung 3 zeigt eine symbolische Darstellung der Regulationsmatrix.

Obwohl es keine allgemein gültigen Regeln zur Ausgestaltung der Regulationsmatrix und - daraus abgeleitet - der Aufgaben des Staates gibt, bietet dieser Ansatz gleichwohl die Möglichkeit der Erfassung und Diskussion des gesellschaftlichen Gesamtzusammenhangs zum Beispiel bei Fragen der Dezentralisierung oder Deregulation. Darüber hinaus ist es möglich, eine exakte Bestandserfassung und Analyse für eine gegebene Situation vorzunehmen, Defizite zu identifizieren und durch Veränderung der horizontalen und vertikalen Regulationsstrukturen alternative Konzepte zu entwickeln.

Insgesamt bleibt festzuhalten, dass der Staat die wirtschaftliche und soziale Entwicklung der Gesellschaft auf vielerlei Weise beeinflussen kann, wobei Höhe und Verwendung der eingetriebenen Monopolrente von entscheidender Bedeutung sind. Einerseits werden den anderen Akteuren Ressourcen entzogen, die diese nicht mehr einsetzen können. Andererseits nimmt die Verwendung der Monopolrente Einfluss darauf, welche Art von Gütern und Dienstleistungen der Staat in welchem Umfang bereitstellen kann. Mit Hilfe des NIÖ-Ansatzes gelingt es also, den Zusammenhang von institutioneller Ordnung oder von gesellschaftlichen Regeln und wirtschaftlicher Entwicklung zum einen schärfer zu fassen als es z.B. soziologischen und ethnologischen Arbeiten gelingt, die sich auch mit der Bedeutung des Wertesystems beschäftigen (vgl. KIEvelitz 1988, OHE et al. 1982). Zum anderen kann das Funktionieren der Gesellschaft (die «Selbstdurchsetzung») anhand von konkreten Organisationsstrukturen beschrieben werden, und es muss nicht auf abstrakte kybernetische Modelle mit komplexen Begrifflichkeiten wie dem der Autopoiesis (Selbstorganisation) von LUHMANN (1984) zurückgegriffen werden (Richter \& Furuboton 1999: 453-454).

\section{Dezentralisierung und NIÖ}

Insbesondere Abb. 3 verdeutlicht, dass Dezentralisierung heute nicht mehr nur als ein Instrument zur Veränderung von administrativen, fiskalischen und politischen Verantwortlichkeiten zugunsten rangniederer staatlicher Organisationen (LITVACK et al. 1998) gesehen werden kann. Das Stichwort der Deregu- 
lation steht für die seit den 80er Jahren verstärkt geführten Debatten um eine Reorganisation der bestehenden, historisch gewachsenen Regulationsrahmen. Insgesamt ist dabei eine allgemeine Tendenz der horizontalen und vertikalen Dezentralisierung im Sinne von Abbildung 3 festzustellen (vgl. Tномі 2001).

In der vertikalen Dimension von Dezentralisierung geht es um die Neudefinition und Reorganisation von Aufgaben innerhalb einer Regulationsform, also z.B. des Staates (z.B. Dezentralisierung), aber auch des Marktes (z.B. Outsourcing, Lean Production). Die horizontale Dimension beinhaltet die Reorganisation der Arbeitsteilung zwischen den Regulationsformen des Staates, des Marktes, der Zivilgesellschaft und der Haushalte. Beispielsweise kann eine bislang von einem zentralstaatlichen Versorgungsunternehmen gewährleistete Wasserversorgung dezentralisiert werden, indem Nutzergemeinschaften oder auch private Unternehmen den Betrieb übernehmen. Vertikale und horizontale Dezentralisierungsprozesse können gleichzeitig stattfinden und sich natürlich auch wechselseitig beeinflussen.

Es stellt sich nun im engeren Kontext der politischen Dezentralisierung die Frage nach der Bedeutung der subnationalen Regulationsrahmen, da es im Zuge von Dezentralisierungsprogrammen oder Maßnahmen stets um eine Stärkung der subnationalen Ebenen geht. Da die Raumwirksamkeit der staatlichen Aktivitäten sich zu einem großen Teil über die territorial organisierte Verwaltungsstruktur vollzieht, erscheint ihre Veränderung zum Zwecke der Effizienzsteigerung als logisch und vor dem Hintergrund der Erfahrung des Scheiterns des zentralen Entwicklungsstaates in vielen Entwicklungsländern (vgl. WuNSCH \& OLuwu 1990) auch als zwingend. Die NIÖ unterstützt diese Sichtweise durch zahlreiche Argumente. Allerdings unterstützte bereits die «alte» oder klassische Institutionenökonomie politische Dezentralisieung mit im wesentlichen zwei Argumentationssträngen:Zum einen ging es um bessere Befriedigung der regional sehr unterschiedlichen Bedürfnisse und Präferenzen der Bürger und zum anderen um die Förderung von Innovationen durch Dezentralisierung, da dezentrale Systeme mehr Lösungsansätze und damit tendenziell bessere Ergebnisse produzieren (vgl. VAUBEL 2001). Mit Hilfe der neuen Institutionenökonomie lassen sich nun zusätzliche Argumente für eine Dezentralisierung finden. VAubel (2001) formuliert insgesamt sechs Punkte, die eine politische Dezentralisierung unterstützen:

- Dezentralisierung senkt durch die größere Nähe zum Bürger dessen politische Informationskosten.

- Dezentralisierung verstärkt die Informationsanreize für Bürger, da Politik zugänglicher und nachvollziehbarer wird sowie die Bedeutung des Einzelnen zunimmt.

- Dezentralisierung verstärkt den Anreiz des Politi- kers, die Bürokratie wirksam zu kontrollieren, da die Wähler ihn für deren Fehlverhalten verantwortlich machen. Auch für die Verwaltung nimmt der Anreiz zu rechtschaffenem Verhalten wegen der größeren Öffentlichkeit zu.

- Mit der Dezentralisierung steigen die Vergleichsmöglichkeiten der Bürger und damit nimmt der Wettbewerb zwischen den lokalen Regierungen ebenso zu, wie die Wahrscheinlichkeit von unsachgemäßer Mittelverwendung abnimmt. In der Konsequenz kommt es zu einem Sinken der Staatsquoten.

- Dezentralisierung schützt regionale Minderheiten vor der relativen Willkür des zentralen Machtapparates und fördert die Berücksichtigung von deren Interessen auch auf Ebene der nationalen Politik.

- Dezentralisierung fördert die Gewaltenteilung und wirkt der Machtkonzentration und den damit für den Bürger verbundenen Risiken entgegen.

Die Perspektive der NIÖ erlaubt nun eine gleichzeitige Thematisierung der technischen und politischen Dimension von Dezentralisierung, indem sie Strukturen (Verwaltungsabläufe) immer auch als Anreizsysteme für die Interessen der betroffenen Akteure sieht. Damit gelingt es, den Doppelcharakter des Instrumentes Dezentralisierung zu erfassen und der mancherorts anklingenden Mystifizierungseuphorie entgegenzuwirken. In dieser Perspektive verursacht eine Veränderung der nationalen Verwaltungsmatrix durch Dezentralisierungsmaßnahmen automatisch Modifikationen in der nationalen Politikmatrix und umgekehrt. Viele Dezentralisierungspläne finden in letzterer ihren strategischen Ausgangspunkt. Damit muss der machtpolitische Hintergrund nicht mehr entdeckt werden, sondern er wird systemimmanent vorausgesetzt und erfasst. Konsequenterweise ergibt sich daraus, dass Dezentralisierung nicht per definitionem gut sein muss: «To debate whether decentralization is good or bad is unproductive and misleading since the impact of decentralization depends on design» (LiTvack et al. 1998: 26).

Es handelt sich um einen kontextgebundenen Prozess der Veränderung im meist staatlichen Regulationsgefüge mit jeweils sehr spezifischen Wirkungen.

Beurteilungsmaßstab aus Sicht der Institutionenökonomie ist die effektive und effiziente Versorgung der Bürger mit öffentlichen Gütern zur Senkung ihrer Transaktionskosten. Die in diesem Zusammenhang nötigen öffentlichen Güter müssen effizient bereitgestellt werden. Die Vermittlung von Grundbildung gehört ebenso dazu, wie eine Verkehrsverbindung, ein Markt oder ein Mindestmaß an Rechtssicherheit und Währungsstabilität. Erst unter diesen Voraussetzungen wird es für die ihren Eigennutz verfolgenden Individuen sinnvoll und möglich, sich in regelmäßigen und komplexeren ökonomischen Transaktionen zu engagieren. Damit ermöglicht die NIÖ die Thematisierung von Entwicklung und Unterentwicklung auf 
der Ebene von Anreizsystemen, wodurch es nicht nur zu neuen und interessanten Analysen und Interpretationen kommt, sondern sich für die Entwicklungszusammenarbeit auch neue Einsichten und Gestaltungsperspektiven ergeben können.

Die NIÖ bietet somit die Möglichkeit, gerade ein so komplexes Feld wie Dezentralisierung mit einem kohärenten Ansatz zu analysieren und zu gestalten.

Es gelingt, die Rolle des Staates und der Verwaltung im Kontext der Regionalentwicklung besser zu thematisieren und damit deren Raum- und Entwicklungswirksamkeit vertiefend erklären zu können. Damit wird der Geographie eine Methodik und ein Instrument geliefert, das präzisere Aussagen über den Zusammenhang von Organisationen und räumlichen Entwicklungen erlaubt, indem es das nationale Abstraktionsniveau der regulationstheoretischen Ansätze ergänzt und die konzeptionelle Erfassung der Meso- und Mikroebenen erlaubt (vgl. BERNDT 1999).

\section{Anmerkung \\ Die hier vorgetragenen Gedanken entstanden im Zusammenhang mit dem von der Deutschen For- schungsgemeinschaft (DFG) geförderten Forschungs- vorhaben «Endogene Potentiale und Dezentralisie- rung». Der Verfasser bedankt sich für die finanzielle Unterstützung.}

\section{Literatur}

Adam, M. (2000): Die Entstehung des GovernanceKonzepts bei Weltbank und UN. Die EZ wird politischer. - In: Finanzierung und Entwicklung 41/10: 272-274.

Agrawal, A. (1999): The Politics of Decentralization A Critical Review. - In: Welt Trends 25: 53-74.

Beier, C. (1995): Dezentralisierung und Entwicklungsmanagement in Indonesien. Beobachtungen zur politisch-administrativen und zur wissenschaftlichen Kommunikation: Ein systemtheoretischer Versuch. = Sozialwissenschaftliche Studien zu internationalen Problemen 198, Saarbrücken:Verlag für Entwicklungspolitik.

BERNDT, C. (1999): Institutionen, Regulation und Geographie. - In: Erdkunde 53/4: 302-316.

BoDemer, K. (Hrsg.) (2000): Subsidiaritätsprinzip, Dezentralisierung und Local Government. Konzeptionelle Fragen und Fallbeispiele aus drei Kontinenten. - = Beiträge des Arbeitskreises «Entwicklung und Verwaltung», Hamburg: Institut für Iberoamerika-Kunde.

Boyer, R. \& Y. Saillard (eds) (1995): Théorie de la Régulation. L'Etat de Savoirs. - Paris: La Decouverte. Conyers, D. (1984): Decentralization and Development: A Review of the Literature. - In: Public Administration and Development 4: 187-197.
Frenkel, M. \& H.R. Hemmer (1999): Grundlagen der Wachstumstheorie. - München: Verlag Vahlen.

Funr, H. (1999): Institutional Change and New Incentive Structures for Development: Can Decentralization and Better Local Government Help? - In: WeltTrends 25:21-51.

HAYEK, F.A. (1973): Law, Legislation and Liberty, Vol.1. - Chicago: University of Chicago Press.

HoltKamp, T. (1993): Dezentralisierung und Partizipation in Ghana. Neue Ansätze zur Regionalentwicklung. - = Freiburger Studien zur Geographischen Entwicklungsforschung 6, Saarbrücken: Verlag Breitenbach.

Kievelitz, U. (1988): Kultur, Entwicklung und die Rolle der Ethnologie. - Bonn: Passagen Verlag.

KRÄTKE, S. (1996): Regulationstheoretische Perspektiven in der Wirtschaftsgeographie. - In: Zeitschrift für Wirtschaftsgeographie 40(1-2): 6-19.

Litvack, J.H., Ahmad, J. \& R. Bird (1998): Rethinking Decentralization in Developing Countries. - (Sector Studies Series), Washington, D.C.: World Bank.

Mueller, D.C. (1995): Public Choice II. A revised Edition of Public Choice. - Cambridge: Cambridge University Press.

Musgrave, R.A. \& P. Musgrave (1973): Public Finance in Theory and Practice. - New York: MacGraw-Hill.

NorTH, D.C. (1992): Institutionen, institutioneller Wandel und Wirtschaftsleistung. - = Die Einheit der Gesellschaftswissenschaften 76, Tübingen: J.C.B. Mohr (Paul Siebeck).

OAtes, W. (1972): Fiscal Federalism. - New York: Harcourt, Brace, Jovanovich.

Ohe, W., Hilmer, R., Nett-Kleybold, S., Esterhasy, Y., \& M.T. KASTL (1982): Die Bedeutung sozio-kultureller Faktoren in der Entwicklungstheorie und -praxis. - = Forschungsberichte des BMZ 29, München.

Richter, R. \& E.G. Furuboton (1999): Neue Institutionenökonomik. Eine Einführung und kritische Würdigung. - (2.Auflage), Tübingen: J.C.B. Mohr (Paul Siebeck).

RondinelLI, D.A. (1999): Dezentralisierung und wirtschaftliche Entwicklung: Eine Einführung. - In: WeltTrends 25: 7-20.

Rondinelli, D.A. \& G.S. Cheema (eds) (1983): Dezentralization and Development. Policy Implementation in Developing Countries. - Beverly Hills: Sage Publications.

Schüller, A. (Hrsg.) (1983): Property Rights und ökonomische Theorie. - München: Verlag Vahlen.

Simon, K. (2000): Local Governance nach dem Subsidiaritätsprinzip in Entwicklungsländern. - In: BODEMER, K. (Hrsg.): Subsidiaritätsprinzip, Dezentralisierung und Local Government. Konzeptionelle Fragen und Fallbeispiele aus drei Kontinenten. - Hamburg: Institut für Iberoamerika-Kunde: 9-28.

Simon, K., Stockmayer, A. \& H. Fuhr (Hrsg.) (1993): Subsidiarität in der Entwicklungszusammenarbeit: Dezentralisierung und Verwaltungsreform zwischen 
Strukturanpassung und Selbsthilfe. - Baden-Baden: Nomos Verlag.

Slater, D. (1989): Territorial Power and the Peripheral State: The Issue of Decentralization. - In: Development and Change 20: 501-512.

Tномі, W. (1999): Nationalstaatliche Regulation und Dezentralisierung: Local Government Reform in Ghana. - In: Welt Trends 25: 99-120.

Tномі, W. (2001): Hoffnungsträger Dezentralisierung. - In: Thomi, W., Steinich, M. \& W. Polte (Hrsg.): Dezentralisierung in Entwicklungsländern. Jüngere Ursachen, Ergebnisse und Perspektiven staatlicher Reformpolitik. - Baden-Baden: Nomos-Verlag: 14-38. ThOMI, W., YANKson, P.W.K. \& S.Y. ZANU (eds) (2000): A Decade of Decentralization in Ghana. Retrospect and Prospects. - Accra: Gold Type.

Thomi, W., Steinich, M. \& W. Polte (Hrsg.) (2001): Dezentralisierung in Entwicklungsländern. Jüngere Ursachen, Ergebnisse und Perspektiven staatlicher Reformpolitik. - Baden-Baden: Nomos Verlag. VAuBEL, R. (2001): Dezentralisierung in Entwicklungsländern aus Sicht der Neuen Institutionenökonomik.In: Thomi, W., Steinich, M. \& W. Polte (Hrsg.): Dezentralisierung in Entwicklungsländern. Jüngere Ursachen, Ergebnisse und Perspektiven staatlicher Reformpolitik. - Baden-Baden: Nomos-Verlag: 56-71.

WORLD BANK (1997): The State in a Changing World. = World Development Report 1997, Washington D.C. Wunsch, J. \& D. Oluwu (eds) (1990): The Failure of the Centralized State. Institutions and Self-Governance in Africa. - San Francisco: IGS Press.

\section{Zusammenfassung: Institutionenökonomische Perspektiven im Kontext der Reorganisation subnationaler Gebietskörperschaften}

Die neue Institutionenökonomie beschäftigt sich auf der Basis eines methodischen Individualismus (Eigennutz der Individuen) mit der Regulation gesellschaftlicher Prozesse durch Normen und Regeln (Institutionen), die das rationale Handeln der Individuen steuern. Regeln und Normen senken die individuellen Transaktionskosten und ermöglichen damit Entwicklung, erfordern aber gleichzeitig zur Aufrechterhaltung ihrer Wirksamkeit Organisationen (Staat etc.) und damit verbunden kollektive Kosten. Der Beitrag bietet aus der Perspektive der Institutionenökonomik einen neuen Interpretationshintergrund für Dezentralisierungsprozesse, stellt einige für die Dezentralisierungsproblematik wichtige Ansätze vor und diskutiert deren Bedeutung für die Analyse und Gestaltung von Dezentralisierungsmaßnahmen.

\section{Summary: Institutional Economics and Decentralisation}

The new institutional economics focuses on the importance of rules and regulations (institutions) for development in general and economic development in particular. An individual's rational choice is directed by rules and regulations which intend to reduce individual (economic) transaction costs. This is regarded as a pre-requisite for development but institutions also create collective costs by the need of having organisations (state etc.) which can control the observance of and the adherence to institutions. Based on an institutional economics perspective this contribution offers a new background for the interpretation of decentralisation by discussing some important paradigms and their implications for the analysis and design of decentralisation measures.

Résumé: Economie des institutions: perspectives dans le contexte de la réorganisation des collectivités territoriales infranationales

En se basant sur un individualisme méthodique (intérêt personnel des individus), la nouvelle économie des institutions s'intéresse à la régulation des processus sociaux par les normes et les règles (institutions) qui guident l'action rationnelle des individus. Ces règles et ces normes font baisser les coûts de transaction individuels et permettent ainsi le développement, mais, pour rester efficaces, elles nécessitent en même temps des organisations (Etat, etc.) et engendrent donc des coûts collectifs. Cette contribution s'appuie sur l'économie des institutions pour offrir un nouveaux cadre d'interprétation des processus de décentralisation; elle présente quelques jalons importants pour la problématique de la décentralisation et discute de leur importance dans l'analyse et la réalisation des mesures de décentralisation.

Prof. Dr. Walter Thomi, Geographisches Institut, Martin-Luther-Universität HALLE-Wittemberg, D-06099 Halle (Saale).

e-mail: thomi@geographie.uni-halle.de

Manuskripteingang/received/rentrée du mansucrit: 7.2.2001

Annahme zum Druck/accepted for publication/ acceptation à l'impression: 17.4.2001 